\title{
GENETICAL AND CYTOLOGICAL PROOF OF SOMATIC ELIMINATION OF THE FOURTH CHROMOSOME IN DROSOPHILA MELANOGASTER
}

\author{
OTTO L. MOHR \\ Anatomical Institute, The University, Oslo, Norway \\ Received April 29, 1931
}

TABLE OF CONTENTS

PAGE

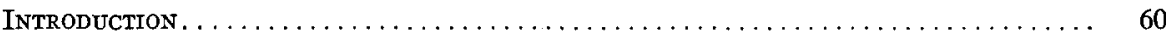

The occurrence and description of the "Minute" mosaic female . . . . . . . . . . . 64

Genetical analysis of the mosaic female . . . . . . . . . . . . . . . . . . . . . . 66

Dominance of the mutant change present in the mosaic . . . . . . . . . . . 66

Inheritance as a member of the fourth chromosome linkage group . . . . . . . . 68

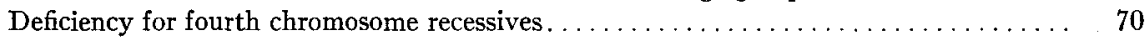

Cytological demonstration of haploidy for the fourth chromosome in the "Minute" flies derived from the mosaic $\ldots \ldots \ldots \ldots \ldots \ldots \ldots \ldots \ldots \ldots \ldots \ldots \ldots \ldots \ldots$

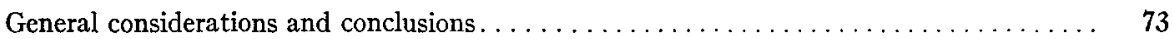

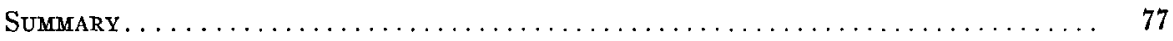

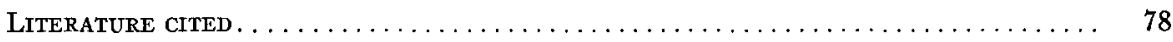

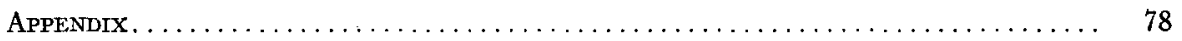

\section{INTRODUCTION}

The general importance of the genetical work in Drosophila melanogaster has to a considerable extent been due to the fact that it has in this material been possible, through detailed experimental analysis of exceptions to normal inheritance, to predict atypical cytological findings, predictions which were found to hold true when the cytological examination was carried out.

Since BRIDGES (1916) by this combined genetical and cytological method delivered the first conclusive proof of the chromosome theory of heredity, a number of cases have been described in which irregularities in the distribution of whole chromosomes during the maturation of the germ cells have led to chromosome aberrations, the nature of which has been cleared up by genetical methods and afterwards verified by cytological examination.

Aberrations from the standard chromosome equipment of the species may, however, also arise through irregularities in the chromosome distribution in somatic cell divisions, giving rise to different types of somatic mosaics.

For a survey of the various types of mosaics that are explainable as due to somatic elimination of one or more chromosomes see a recent paper of L. V. Morgan (1929). Here only the following should be stated: 
By far the most common type of somatic mosaics are the sex mosaics (gynandromorphs). As shown by MORGAN and BRIDGES (1919) the large majority of these gynandromorphs are due to somatic elimination in one of the early cleavage divisions of one member of the $\mathrm{X}$ chromosome pair. The individual starts as a female, but when one $\mathrm{X}$ is eliminated a daughter cell with a single $\mathrm{X}$ chromosome is formed. All those cells which are derived from this daughter cell will be $\mathrm{X} \mathrm{O}$ and male, while the rest of the individual will be $\mathrm{X} \mathrm{X}$ and female. The result is accordingly a gynandromorph consisting of one female and one male part. In most cases these gynandromorphs are more or less bilateral, but not infrequently the female part is larger than the male region.

Principally analogous to these sex mosaics are the so-called Diminished mosaics in which the body is divided into a normal part and a part showing the Dominant character complex Diminished. As shown by BRIDGes (1921) the Diminished character is not due to an ordinary gene, but to haploidy for the small round, so-called fourth chromosome. The Diminished mosaics have correspondingly been interpreted by BRIDGES as $2 \mathrm{~N}$ in the normal and $2 \mathrm{~N}-\mathrm{IV}$ in the Diminished region, due to somatic elimination in one of the early cleavage cells of one member of the fourth chromosome pair (Morgan, BRIDGes, and SturTeVANT 1925).

In Drosophila melanogaster BRIDGes has encountered about a dozen such mosaics, of which the majority were interpreted as IV IV in the normal and IV O in the Diminished part. But the possibility could not be excluded that some of them at any rate, were in reality due to a somatic mutation to a dominant Minute, since the Diminished character complex is strikingly like the character changes typical of the large group of dominant Minute mutations.

In his analysis of the claret mutant type of Drosophila simulans, STuRTEVANT (1929) obtained no less than 171 Diminished mosaics of analogous type. This extraordinarily high number is due to the very peculiar action of the claret gene in homozygous condition. The gene causes irregularities in the chromosome distribution, not only during the maturation of the germ cells, but also in the early cleavage stages of the next generation, resulting in the production of a large number of non-disjunctional exceptions and gynandromorphs as well as of Diminished (haplo-IV) individuals (40 percent of all the offspring) and Diminished mosaics (4 percent of the total offspring). StURTEVANT obtained offspring from three Diminished mosaics, but these included no Diminished individuals.

There are also in Drosophila melanogaster a few cases in which particular genotypic changes increase the frequency of somatic elimination. Thus, 
BRIDGES (1925) has described a sex-linked mutant Minute-n which causes a high percentage of elimination of the Minute-n X chromosome in somatic cells. And Stern (1927a) found that three different III-chromosome Minute mutations caused a high frequency of somatic elimination of particular sections of the third chromosome. In these cases the elimination occurs not in the cleavage cells but during the later larval developmental stages. Only a small part of the individual will accordingly be derived from the atypical cell, and the corresponding somatic alteration will be restricted to a limited region, frequently a mere spot in the surface area of the adult fly.

The genetic analysis of the mosaics resulting from different types of somatic elimination rests on the fact that each part of an individual develops according to its own genetical constitution (MORGAN and BRIDGES 1919).* Thus recessive genes present in the single $X$ chromosome of a gynandromorph manifest themselves somatically in the male part. Haploidy for the fourth chromosome in one region of an individual produces the somatic change Diminished in this region, etc.

By taking advantage of this fact, and by using (or intentionally introducing) "marked" chromosomes containing different mutant genes it has been possible to carry out an analysis that is in most cases fully conclusive, in spite of the fact that the analysis primarily rests on the external inspection of the mosaic without crucial breeding test and cytological control.

As regards those gynandromorphs which are explained by simple single elimination, half of these can also be explained as derived from binucleated eggs. But there is here special genetical evidence in favor of the former explanation. Thus, gynandromorphs with apparently normal male abdomen are always sterile, like the $\mathrm{X} \mathrm{O}$ males due to primary non-disjunction (MORGAN and BRIDGES 1919); the sex-linked bobbed character, which is suppressed in ordinary $\mathrm{X} \mathrm{Y}$ males through the action of the normal allelomorph of bobbed present in the $Y$ chromosome, manifests itself in the male region of gynandromorphs as it does in non-disjunctional X O males of bobbed stock (STERN 1927b); and in a large experiment which was planned in such a way that the chances of obtaining flies positively diagnostic for a binuclear origin were increased by the use of marked autosomes, not a single such fly was obtained, although the experiment produced 12 gynandromorphs that were explainable by simple elimination (L. V. MoRgan 1929).

* For certain exceptions to this rule see a recent paper of DobZeansky (1931). 
Cytological evidence in favor of the elimination explanation was obtained by PEARSon (1927) in gynandromorphs of the grasshopper Amblycorypha. The gonads contained both ovarian and testicular tissue, and the ovarian cells had two $\mathrm{X}$ chromosomes, while the spermatogonies had only one $\mathrm{X}$.

The reasons why it has not, in mosaicism due to somatic elimination, been possible to deliver the complete documentation by combined breeding test and cytological demonstration are different:

On the one hand there is in Drosophila for technical reasons no chance of obtaining cell division figures from both regions of an adult mosaic, and mosaics are not detected in the larval or pupal stages; on the other hand the elimination of a chromosome only in particular cases leads to fertile chromosome combinations; even then the crucial breeding test can, of course, only be carried out if the gonads, or part of the gonads, happen to be included within the atypical and fertile part.

The mosaics which might be expected to offer the best chance of securing the combined genetical and cytological proof of somatic elimination are accordingly those which consist of one $2 \mathrm{~N}$ and one $2 \mathrm{~N}-1$ region, and in which the elimination has occurred so early during the cleavage stages that part of the germinal tissue is derived from that cell which has lost one chromosome by elimination. In this case the atypical part, if fertile, will produce germ cells, half of which will be $\mathrm{N}-1$. When these are fertilized by (or fertilize) normal $\mathrm{N}$ germ cells, the result will be $2 \mathrm{~N}-1$ individuals whose atypical chromosome equipment may be analyzed by appropriate genetical analysis, and of which there will be available a sufficient material for cytological examination.

The commonest type of somatic mosaics, namely, the ordinary gynandromorphs, do not fulfill the above requirements. In the Drosophila gynandromorphs both gonads are in most cases the same, either ovaries or testes. If the genital apparatus is female the gynandromorph, if fertile, breeds as an ordinary female. If the gonads and external genitalia are male, then the gynandromorph is completely sterile.

As regards those mosaics which might be expected to be produced by somatic elimination of the second or third chromosome, no such have ever been encountered, nor have haplo-II or haplo-III individuals been reported from any experiment. Obviously, the lack of one member of the second or third chromosome pair causes such gross changes that cells of this type are unable to develop.

There remains, however, one type of somatic mosaics which, under favorable conditions, may fulfill the requirements outlined above, namely, 
the Diminished (haplo-IV) mosaics. If in a Diminished mosaic the elimination of one member of the fourth chromosome pair has occurred so early during the cleavage stages that part of the gonads are derived from the haplo-IV daughter cell, then there is the chance of obtaining offspring from this haplo-IV part. There is reason to assume that this part will be fertile, since we know from the Diminished individuals that the $2 \mathrm{~N}-\mathrm{IV}$ combination is a fertile combination.

Half of the germ cells from the haplo-IV region of such a mosaic will be functioning nullo-IV germ cells, and when these meet normal haploid germ cells, the result will be Diminished, haplo-IV individuals in which the lack of one member of the fourth chromosome pair may be demonstrated by genetical methods and by cytological examination as well.

It was the latter situation that was realized in the case to be described in detail below.

A preliminary account of this analysis was read before Det NoRSke Videnskapsakademi i Oslo (Mohr 1930).

THE OCCURRENCE AND DESCRIPTION OF THE

"MINUTE" MOSAIC FEMALE

During the analysis of a new III-chromosome dominant mutant Vein $(V)$, heterozygous females which in one member of the third chromosome pair carried roughoid $\left(r_{u}, 0.0\right)$ and Vein, while the other member was wildtype, were mated singly to males homozygous for the following third chromosome recessives: roughoid, hairy $(h, 26.5)$, thread $\left(t_{h}, 42.2\right)$, scarlet $\left(s_{t}, 44.0\right)$, curled $\left(c_{u}, 50.0\right)$, stripe $\left(s_{r}, 62.0\right)$, sooty $\left(e^{s}, 70.7\right)$, and claret $\left(c_{a}, 100.7\right)$. One of these cultures (C.4709, March 6, 1930) gave the following offspring: roughoid Vein 62, wild-type 55, roughoid 10 and Vein 7.

In the wild-type class a single individual was detected (March 12,1930) which was a typical bilateral mosaic, consisting of a normal right half and a left half which had distinctly shorter and more slender bristles and hairs, typically like those present in the different Minute mutations. The left wing was in addition shorter and more bluntly rounded than the normal right wing (figure 1). The exceptional individual was in other respects a typical female; judging from the slightly swollen abdomen, there was reason to believe that she had already been fertilized.

In the head the border line separating the Minute and the wild-type region followed the median line strictly, both on the dorsal and on the ventral surface. This could easily be controlled by aid of the head bristles and larger hairs which were without exception Minute on the left and wildtype on the right side. The eyes were of about equal size, but the left 
antenna and arista, as well as the left half of the distal surface of the proboscis, were smaller on the left than on the right side.

Also on the thorax the border line followed the median line strictly both on the dorsal and on the ventral side, all the bristles and larger hairs, including the sterno-pleurals, being Minute on the left and wild-type on

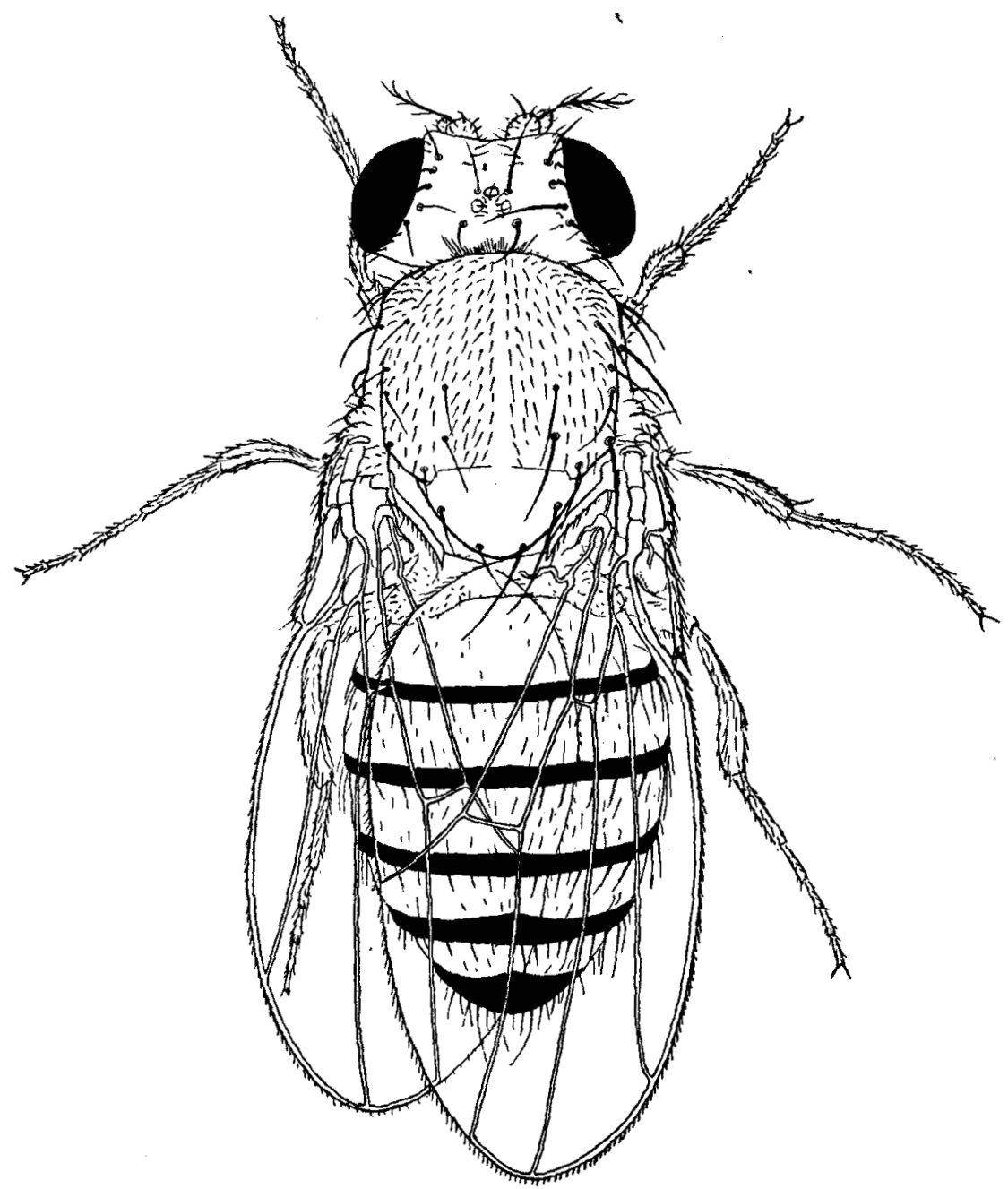

FigURE 1.-Mosaic female. Left half "Minute," right half wild-type.

the right side. Of the two somewhat larger hairs situated just anterior to the second pair of legs on each side of the median ventral furrow, the left was Minute and the right wild-type. The left wing was markedly shorter than the right, bluntly rounded and with a shortened inter-crossvein dis- 
tance. The pair of bristle-like hairs just anterior to the distal costal break were Minute on the left and normal-sized on the right wing.

In the legs there was a slight deviation from the strictly bilateral distribution of the two regions in so far as the larger hairs on the ventral surface of the coxa seemed to be of equal size on both forelegs. From comparison of these hairs in the fixed specimen with the corresponding hairs in wild-type and in Diminished individuals (see p. 64) it was concluded that these hairs were in the mosaic wild-type on both sides. That the rest of the left foreleg was Minute, in contrast to the right foreleg, could be ascertained by comparison of the size of the larger hairs on the femur and the apical and preapical bristles of the tibia. A corresponding difference in size was evident also in the second and third pair of legs and especially striking in the apical and preapical bristles on the tibia of the second pair of legs where they are largest.

In the abdomen the border line separating the Minute and the wildtype region followed strictly the median line on the dorsal surface of the anterior four segments (tergites). This could be ascertained by the difference in size of the larger hairs along the posterior margin of each tergite. The fifth tergite had, in contrast to the others, wild-type hairs both on the left and on the right side. In the remaining part of the abdomen, including the surroundings of the genital and anal openings, the hairs were Minute on the left and wild-type on the right side of the dorsal surface.

On the ventral surface of the abdomen it was evident that the border line followed the median plane strictly, the hairs on the ventral plates being Minute on the left and wild-type on the right side. As regards the anal and genital region it was, by the smallness of the hairs, rather difficult to detect a conclusive difference in size on the ventral side. But after scrutinous examination of the fixed specimen and comparison with wild-type and Diminished flies there seemed to be little doubt that the division in a left Minute and a right wild-type half holds true also for this part of the fly.

Summing up, we find accordingly that we are dealing with a bilateral somatic mosaic in which the division in a left Minute and a right wildtype region is remarkably schematical, the only deviations from the strictly bilateral symmetry being found in the ventral surface of the anterior coxae and in the fifth tergite which are wild-type on both sides.

GENETICAL ANALYSIS OF THE MOSAIC FEMALE

Dominance of the mutant change present in the mosaic

The most plausible explanation of the mosaic female described seemed to be that we were dealing with a somatic mutation to a dominant Minute which had occurred in one of the earliest (probably the first) cleavage 
cells, since dominant Minute mutations are of very frequent occurrence in Drosophila melanogaster. But another possibility, namely, that the Minute region might be haplo-IV due to somatic elimination of one member of the fourth chromosome pair, was also kept in mind.

Since the mosaic was a normal female with normal external genitalia it was hoped that she might prove fertile. And in view of the bilateral distribution of the Minute and wild-type parts it seemed not unlikely that one ovary, or part of one ovary, might be included within the Minute region. In this case the two alternative hypotheses might be put to a test.

The mosaic was accordingly as a first step backcrossed to $r_{u} h t_{h} s_{t} c_{u}$ $s_{r} e^{s} c_{a}$ males (C. 4732). By this mating we would not only certify genetically that the mosaic was derived from C. 4709 and that no contamination had occurred, but we would also obtain information as to the possible linkage relations of the new mutant to genes distributed over the entire third chromosome.

The mosaic proved fertile, but no Minute flies were obtained during the first nine days' counts. Since, however, delayed emergence is a typical feature of the Minute character complex, the counting was continued, and on the tenth day 3 typically Minute individuals were found. The counting was now continued until all the offspring from C.4732 had hatched. In all 262 individuals were obtained, 16 of which were Minute.

Meanwhile the mosaic female had already on the sixth day been transferred into a new culture bottle (C.4732 a) with the same males, and in this culture the offspring were counted for the ordinary ten days only. The mosaic female which was still vigorous was now drawn and afterwards fixed in alcohol for later examination. The total output of C.4732a were 131 individuals, of which 15 were Minute. In both cultures more than twothirds of the Minute flies were males, thus indicating that the Minute females had a lower viability than the Minute males in these cultures.

In the following are given the total output of the two cultures, the numbers from C. 4732 being printed in ordinary letters, those from C. $4732 \mathrm{a}$ in italics. The numbers in parenthesis denote the Minute individuals out of the total in the respective class:

$r_{u} h t_{h} s_{t} c_{u} s_{r} e^{s} c_{a} 21,19$ (2), wild-type $52(6), 26(1) ; r_{u} 17$ (2), $7(1), h$ $t_{h} s_{t} c_{u} s_{r} e^{s} c_{a} 14,4 ; r_{u} h 5(1), 8(2), t_{h} s_{t} c_{u} s_{r} e^{s} c_{a} 8,6 ; r_{u} h t_{h} 2, s_{t} c_{u} s_{r}$ $e^{s} c_{a} 2 ; r_{u} h t_{h} s_{t} 2, c_{u} s_{r} e^{s} c_{a} 3 ; r_{u} h t_{h} s_{t} c_{u} 6,5 ; s_{r} e^{s} c_{a} 16(2), 3(1) ; r_{u} h$ $\dot{t}_{h} s_{t} c_{u} s_{r} 4,3, e^{s} c_{a} 2,1 ; r_{u} h t_{h} s_{t} c_{u} s_{r} e^{s} 9,8, c_{a} 32(3), 11(3) ; r_{u} s_{r} e^{s} c_{a} 4$, $h t_{h} s_{t} c_{u} 4 ; r_{u} e^{s} c_{a} 3,1, h t_{h} s_{t} c_{u} s_{r} 1 ; r_{u} c_{a} 10$ (1), 9 (4), $h t_{h} s_{t} c_{u} s_{r} e^{s} 12,2$; $t_{h} s_{t} 2 ; r_{u} h s_{r} e^{s} c_{a} 6, t_{h} s_{t} c_{u} 2 ; r_{u} h e^{s} c_{a} 1, t_{h} s_{t} c_{u} s_{r} 2 ; r_{u} h c_{a} 4,7, t_{h} s_{t} c_{u} s_{r}$ $e^{s} 9,2 ; r_{u} h t_{h} s_{r} e^{s} c_{a} 1, s_{t} c_{u} 1 ; s_{t} c_{u} s_{r} 1(1) ; r_{u} h t_{h} s_{t} e^{s} c_{a} 1, c_{u} s_{r} 1 ; r_{u} h$ 
$t_{h} s_{t} c_{a} 1, c_{u} s_{r} e^{s} 2 ; r_{u} h s_{t} c_{u} c_{a} 1, s_{r} e^{s} 1,1 ; h s_{r} e^{s} c_{a} 1 ; r_{u} c_{u} s_{r} e^{s} 1 ; r_{u} s_{r} e^{s}$ $1(1) ; r_{u} e^{s} 1 ; t_{h} s_{t} c_{u} c_{a} 1$.

From this result it is apparent that the supposedly non-virgin mosaic female has at any rate not been fertilized by a Vein brother, since none of her sons and daughters were Vein. Whether she has been fertilized by a wild-type brother is difficult to tell with certainty. The marked numerical preponderance of wild-type non-crossovers in C. 4732 as compared with the corresponding non-crossover class might seem to point in this direction. But an analogous discrepancy is also seen in the two single crossover classes resulting from crossing over within the $e^{s}-c_{a}$ region, so it is quite possible that the numerical difference is simply due to the markedly lowered viability in this culture of those flies which are homozygous for roughoid and in addition for the largest number of the other third chromosome recessives present in the test.

Another source of error should also be kept in mind, namely, the possibility of overlapping of generations in C. 4732 where the offspring were counted for 19 days. Thus some of the apparent triple crossovers in this culture may be due to overlapping. A calculation of the linkage values for the different III-chromsome mutant genes involved in the backcross is of no particular interest for our present purpose. In spite of the abovementioned sources of error such a calculation based on the total data gives values which are in sufficient accordance with the known map distances to show that crossing over has been of normal frequency throughout the third chromosome in this test.

The reappearance of Minute flies in $F_{1}$, flies which in every respect resembled the Minute half of the mosaic mother, proved conclusively that at any rate part of the gonads have been included within the mutated part of the mosaic, and, furthermore, that the genotypical change responsible for the Minute character complex here dealt with is inherited as a typical Mendelian dominant.

If, which seems likely, one ovary is included in the mutated region, then we would expect one quarter among the offspring of the mosaic to be $\mathrm{Mi}$ nute (see p. 67), while here only 31 Minutes were obtained as against 362 not-Minute individuals. That this deviation from the expectation might simply be due to the lowered viability of the Minute phenotype was not unlikely since the Minute individuals were examined and found to be small and weak looking in comparison with their non-Minute sibs.

Inheritance as a member of the fourth chromosome linkage group

From the distribution of the Minute individuals in the different crossover classes in the above test, with preferential occurrence in the most 
viable classes, it was clear that the new mutant did not belong to the IIIchromosome linkage group. And by examination of the Minute individuals it was found that they resembled closely the Diminished, haplo-IV flies described by BRIDGES (1921): They were in comparison with the wildtype of a smaller size throughout, had shorter and more slender bristles, a paler body color, a darker trident pattern on the thorax, larger eyes, and wings which were frequently slightly divergent, blunter and less clear in texture. This in combination with the delayed emergence and the lowered viability made it at once probable that we were dealing not with an ordinary dominant Minute mutation but with individuals which had the fourth chromosome in haploid condition.

Minute males from C. 4732 which were heterozygous for the abovementioned III-chromosome recessives had in the meantime been mated to a homozygous roughoid female (C. 4772). The result of this mating was roughoid 26, wild-type 29 , roughoid Minute 9, Minute 13 . Since there is no crossing over in the males, this result confirmed the above conclusion that the Minute mutant did not belong to the third chromosome linkage group. And since there were both females and males in the Minute classes this test proved simultaneously that the Minute mutant did not belong to the first (the sex) chromosome.

In order to test whether the mutant belonged to the II-chromosome group Minute males were also mated to females homozygous for the IIchromosome recessives black $(b)$ purple $\left(p_{r}\right)$ and curved $(c)$, and $F_{1}$ Minute males backcrossed to black purple curved females (C.4798-99). The result of this male backcross was: $b p_{r} c 192$, wild-type 232, $b p_{r} c$ Minute 5 , Minute 69 . This test proves that Minute does not belong to the second chromosome group of linked characters and gives further evidence of the heavy mortality of the Minute flies. This lowering of the viability was particularly extreme in Minute flies which were at the same time homozygous for black purple and curved, a combination which in itself reduces the viability of the fly somewhat (see on this point p. 68).

When it had been proved that Minute showed free segregation with representatives of the first, second and third chromosome linkage groups, it was clear that the dominant character was due to a change in the fourth chromosome since there are in Drosophila melanogaster four chromosome pairs only.

This change might either be a dominant mutant gene or lack of one member of the fourth chromosome pair. The latter possibility seemed the more probable in view of the phenotypical similarity of the Minute here studied with Diminished. Additional evidence in the same direction was 
also derived from a study of the fertility of the Minute flies. While the Minute males as a rule proved to be of fairly good fertility, sterility or lowered productivity was a typical feature of the Minute females. Among 12 preliminary test cultures where one or (in some cases) more Minute females were mated to unrelated males only six delivered a limited amount of offspring. In larger mass cultures, however, a fair amount of offspring from Minute females could generally be obtained. A Minute stock was therefore maintained by mating Minute flies in mass cultures. It was, however, found advisable to add one or two wild-type sisters in order to get the stock started. An analogous lowering of the fertility is typical also of the Diminished, haplo-IV females.

\section{Deficiency for fourth chromosome recessives}

Whether the Minute character change studied was due to a dominant fourth chromosome gene or to haploidy for the fourth chromosome could easily be tested by mating the Minute flies to flies homozygous for the fourth chromosome recessives bent and eyeless, the only representatives of the fourth chromosome group of which stocks were at disposal in the laboratory. As shown by BRIDGES (1917) recessive genes located in the region opposite to a deficient chromosome section manifest themselves in heterozygous condition in flies carrying the deficiency, since their normal allelomorphs are lost or inactivated. If the Minute individuals are deficient for one member of the fourth chromosome pair, we expect accordingly that bent and eyeless will manifest themselves in $F_{1}$ of Minute $\times$ bent and Minute $X$ eyeless respectively.

Females homozygous for black ( $b$, II chromosome) pink ( $p$, III chromosome) and bent ( $b_{t}$, IV chromosome) were in 10 cultures mated to Minute males [C. 4808 ( $2 \%$ \%) $4831-32,4843,4860-66$ ].1 The total output of these cultures were 901 wild-type individuals while only a single Minute male hatched. The latter fly showed the bent character in very extreme form; the wings were folded and the tarsi very much shortened and fused together so that their hairs formed a thick, dark brush distal to the shortened tibia.

1 One such mating (C. 4809) gave an entirely unexpected result, namely, wild-type $37 \& q$, $8 \sigma^{7} \sigma^{7}$; bent $14 \% \&, 4 \sigma^{7} \sigma^{7}$. The bent character of these $F_{1}$ non-Minute bent flies was very well pronounced, much more so than is the case in the $b p b_{i}$ stock flies, where the bent character is poorly developed. When inbred, these $F_{1} b_{t}$ flies behaved as ordinary homozygous $b_{t}$. If the Minute type is haplo-IV, as later proved to be the case, the simplest explanation of the above result is the assumption that the $\mathrm{P}_{1} b p b_{t}$ female has been triplo-IV $\left(b_{t} b_{t} b_{t}\right)$. When mated to haplo-IV males the expectation is then: wild-type triplo-IV $\left(b_{t} b_{t}+\right)$, wild-type heterozygous for $b_{t}\left(b_{t}+\right)$, homozygous $b_{t}\left(b_{t} b_{t}\right)$ and Minute-bent haplo-IV $\left(b_{t}\right)$ individuals in equal numbers. The latter type is inviable (see p. 71). Due to extraneous causes the appropriate tests had to be postponed, and in 
From this result it was clear that the viability of the $\mathbf{F}_{\mathbf{1}}$ Minute-bent compound was lowered almost to complete lethality, a fact which was also apparent from the dead pupae present in these cultures. When dissected they were found to contain crippled Minute-bent individuals. By aid of the shortened legs with their dark, hairy brush it was also possible to recognize the Minute-bent compound flies through the wall of the puparium. And from all but two of the above cultures Minute-bent flies that were still living, 21 in all, were as a control secured by dissection.

The analogous matings of eyeless female $\times$ Minute males (C. 4810-12) had a similar result. In all 482 wild-type flies were obtained as against 13 Minute-eyeless compound individuals $\left(3 \% q, 10 \sigma^{x} \sigma^{x}\right)$. Thus, also, here the viability of the compound is very markedly lowered. The eyeless character in the Minute-eyeless flies that hatched was not conspicuously more extreme than in ordinary eyeless stock flies. But in individuals that were secured by dissection of Minute-eyeless pupae the eyes seemed on the whole to be slightly smaller than in ordinary eyeless, although the difference was rather inconspicuous here also.

In order to compare the character changes in the Minute-eyeless compound with ordinary eyeless under optimal conditions, Minute-eyeless compound males from C.4811 were mated to eyeless females from stock (C. 4830, 4870). In one of these cultures where a single eyeless female was used 123 eyeless and 6 Minute-eyeless $\left(\sigma^{7} \sigma^{\top}\right)$ were obtained; in the other with three $\mathrm{P}_{1}$ eyeless females, the output was 251 eyeless and 27 Minuteeyeless $\left(6\right.$ o $\left.~ o, 21 \sigma^{7} \sigma^{7}\right)$. The eyes were in the Minute-eyeless compound, on the whole not conspicuously smaller than in their ordinary eyeless sibs. In C.4830, however, some eyeless individuals occurred that had such large eyes that they overlapped wild-type so that their homozygous eyeless genotype had to be controlled by testing; no such overlapping individuals were encountered among the Minute-eyeless flies, a fact which involves indirect evidence to the effect that the eyeless character is more pro-

order to secure a stock for later examination the non- $b_{t}$ flies were searched for individuals which showed the (slight) dominant characteristics described by BRIDGes as typical of the triplo-IV type. It was believed that such were found, and by selection a stock was maintained which continued to throw a percentage of flies with slightly shortened and coarser bristles. When one such fly (third generation) in a mating to Minute (haplo-IV) gave non-Minute bent in $F_{1}$, it was believed that the bristle alteration was a reliable somatic indication of the triplo-IV condition. However, when the case was taken up later, it turned out that the bristle alteration mentioned was due to a second chromosome recessive which overlapped the wild-type, and the opportunity to prove the case had accordingly been lost.

The atypical sex ratio in the above culture was found to be due to two sex-linked lethals for which the $P_{1}$ female had been heterozygous, one located in the close neighborhood of vermilion and the other about 10 units to the right of forked.

Genetres 17: Ja 1932 
nounced in the compound. That the exaggeration of eyeless is not more distinct may seem natural when it is remembered that the Minute flies have larger eyes than the wild type. The Minute character in other words counteracts the tendency to reduction of the size of the eyes. Still, the very slight exaggeration of the eyeless character contrasts somewhat with other typical cases of exaggeration and may need further investigation (see also p. 80).

The tests presented above proved conclusively that the dominant $\mathrm{Mi}$ nute here dealt with was not due to a point mutation, but to a deficiency which includes the fourth chromosome loci bent and eyeless. The dominance (of Minute) and the exaggeration of the included characters (bent and eyeless) are general deficiency phenomena typical of section deficiency (MOHR 1919) and quite similarly of chromosome deficiency (BRIDGES 1921).

The above results were in all essential respects parallel to those obtained by BRIDGEs in matings of Diminished (haplo-IV) to bent and eyeless. As regards the very marked lowering of the viability of the compounds, by BRIDGES considered as another expression of the exaggeration, the following should be added:

Based on the tests presented above (C.4772, 4798-99, p. 69) the viability of Minute as compared with the wild type is 31.4 percent (261 wildtype and 82 Minute, where equality is expected). In some later special matings of wild-type female $\times$ Minute males (C. 4953-56) the result was wild-type 421, Minute 160 (67 $\circ$ \& , $\left.93 \sigma^{7} \sigma^{7}\right)$, that is, the viability of Minute is here 38 percent. To these data may be added those derived from the experiments p. 78 and p. 79 , in the majority of which the viability of Minute was far better (C. 4959, 4975, 4977, 5038, 5051-52). These tests comprise 489 wild-type and 353 Minute individuals, where equality is expected, corresponding to a viability of 72.2 percent.

The total data comprise 1171 wild-type as against 595 Minute individuals, that is, the average viability of Minute is 50.8 percent, a value which is strikingly in accordance with that found by BRIDGEs for Diminished, namely, 53 percent. But it is evident that the viability of Minute is exceedingly variable, probably as a consequence of slight differences in the culture conditions. In the tests here presented the viability ranges from 22-24 percent (C. 4959, 4798, p. 78) to cases in which the Minute class even outweighs the corresponding wild-type class (C. 5038, 5052, p. 79).

Based on the data presented above (p. 70) the viability of the Minutebent compound is practically zero as compared with the wild type, while that of the Minute-eyeless compound is 2.7 percent. In analogous tests 
with Diminished (haplo-IV) BRIDGes found the corresponding values to be 0.5 and 1.3 percent respectively. As compared with homozygous eyeless, which by itself has a slightly lowered viability, the viability of the Minute-eyeless compound was 8.8 percent (C. 4830,4870, p. 71). This disproportionate lowering of the viability of the compounds is evidence of exaggeration of the included characters.

Finally, the homozygous lethal action of the Minute dealt with was further evidence of deficiency. Not only was it impossible to reduce the number of wild-type flies by continuous inbreeding, but all the Minute individuals used in the numerous crosses proved to be heterozygous only. It was accordingly regarded unneccessary to control this point further by special tests.

Strictly speaking the above tests demonstrate that the Minute flies are deficient for a section of the fourth chromosome including the loci for bent and eyeless. But in view of the striking similarity with Diminished, not only in somatic, but also in all genetical and in physiological respects (lowered viability, lowered fertility, late emergence, etc.), it was now practically certain that we were not dealing with a section deficiency but with a chromosome deficiency involving one member of the fourth chromosome pair. This could easily be controlled by cytological examination of cells from the Minute individuals.

CYTOLOGICAL DEMONSTRATION OF HAPLOIDY FOR THE FOURTH CHROMOSOME IN THE "MINUTE" FLIES DERIVED FROM THE MOSAIC

Ovaries of Minute females were fixed in strong Flemming's solution and colored with iron-haematoxylin in the ordinary way. A number of satisfactory oogonial plates were found in eight different Minute females, and all had only one small spheric "fourth" chromosome, as had been predicted. Representative photographs and drawings of oogonial plates from four different females are presented in figure 2 and need no further comment. As will be seen, all the other chromosomes are present in duplicate as usual. The result of the cytological examination proves that our Minute mutation is identical with the Diminished, haplo-IV mutant type.

GENERAL CONSIDERATIONS AND CONCLUSIONS

The evidence presented above proves conclusively that the left half of the original mosaic was haplo-IV. Hence, it has in this case been possible to deliver the complete proof that the haplo-IV condition has arisen through somatic elimination resulting in mosaicism. Though the earlier special genetic evidence of somatic elimination of one member of a chro- 
mosome pair is in Drosophila fully convincing (see Introduction) the present case fills a gap in so far as it represents the first in which the elimination of a chromosome in somatic cells has been proved by complete breeding tests and cytological demonstration. Thus, it puts the proof of somatic elimination and mosaicism on the same level as those previously delivered for chromosome aberrations due to non-disjunction during the maturation of the germ cells. As regards some of the earlier mosaics which were interpreted as Diminished (haplo-IV) the possibility could not be excluded

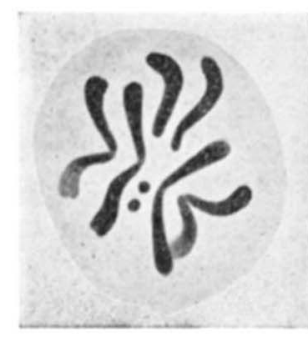

a

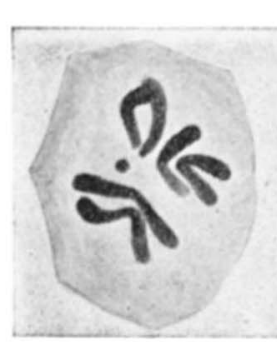

e

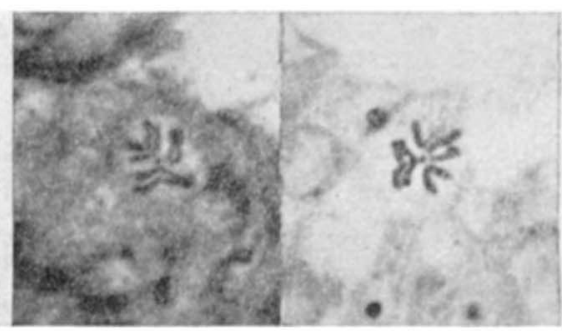

b c

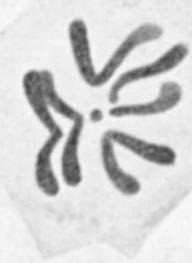

g

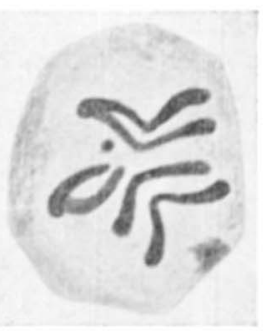

d

FiguRE 2.-Oogonial plate from 2n Drosophila melanogaster female, for comparison. b-h oogonial plates from five different Diminished (haplo-IV) females derived from the mosaic. Note: One small round fourth chromosome only; b and c photographs, about $2000 \times ;^{2} \mathrm{~d}-\mathrm{h}$ drawings, Zeiss Comp. Oc. 12, Obj. $1.5 \mathrm{~mm}$ Apochromat N.A. 1.30, drawn at table level by aid of Abbés apparatus, Tube length $160 \mathrm{~mm}$; $f$ same as b; g same as c.

Strong Flemming's solution; iron-haematoxylin.

that they were due to somatic mutation to a dominant Minute in one of the early cleavage cells. This source of error is here eliminated.

The bilateral symmetry of the mosaic studied makes it practically certain that the elimination of one member of the fourth chromosome pair has occurred during the first cleavage division. The slight deviations from the

${ }^{2}$ For the preparation represented in figures $\mathrm{b}$ and $\mathrm{f}$ as well as for the photographs the author is indebted to kand. real. T. QUELPRUD. 
bilateral symmetry (coxa of first leg, fifth tergite) are due to wandering of the cleavage cells during the developmental stages prior to the formation of the imaginal discs (compare the detailed analysis of the distribution of parts in sex mosaics by STURTEVANr 1929).

The genetical test of the mosaic proves that one ovary (or part of one ovary) is derived from one cleavage cell while the rest of the germinal tissue is derived from another. Hence the germinal tract in Drosophila cannot be derived from one and the same cleavage nucleus. In this respect the

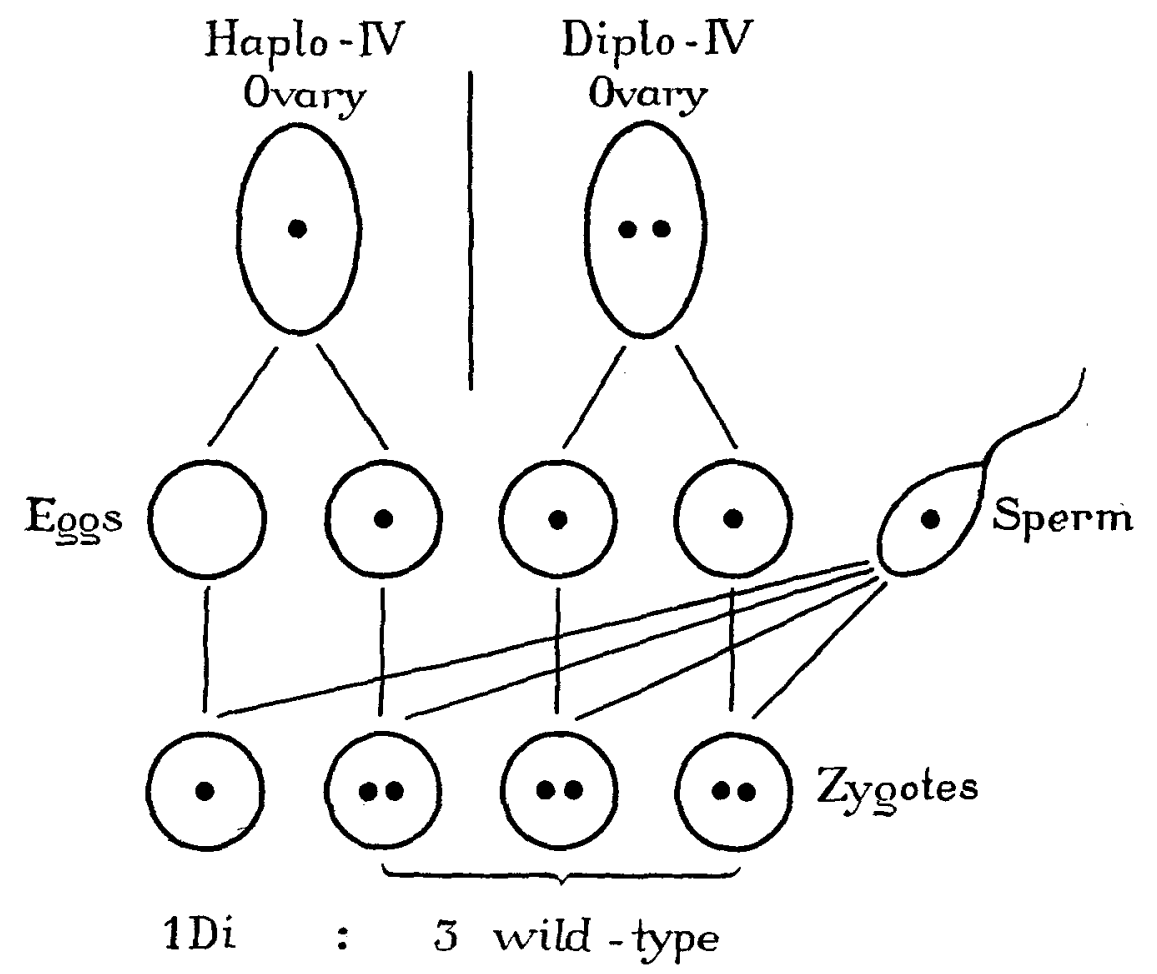

FIGURE 3.- The result of mating the Diminished mosaic to normal males, if one entire ovary is haplo-IV and the other diplo-IV.

genetical evidence is parallel to that obtained in the analysis of a somatic mosaic (MOHR 1923) and in accordance with HuETTNERs (1923) cytological findings that more than one cleavage nucleus gives rise to the primordial germ cells.

In this connection it is of interest to decide whether one entire ovary or only part of an ovary has been included within the haplo-IV part of the mosaic. If one entire ovary is included, we expect half of the eggs from this GeNeTICS I7: Ja 1932 
ovary to be nullo-IV and the other half haplo-IV. The other ovary will deliver haplo-IV eggs only.

Hence if both ovaries deliver an equal amount of eggs, we expect, when these eggs are fertilized by normal, haploid sperms, to obtain non-Diminished and Diminished offspring in a 3:1 ratio (see figure 3). But this expectation will only be fulfilled if the viability of Diminished is on a par with that of the non-Diminished type.

In the test of the mosaic we obtained 362 non-Diminished and $31 \mathrm{Di}$ minished flies. One third of the non-Diminished individuals are on the above assumption derived from the haplo-IV ovary. This ovary has accordingly delivered 121 non-Diminished and 31 Diminished flies. This deviation from the expected $1: 1$ ratio is readily explained by the markedly lowered viability of the Diminished phenotype. Based on the above data the viability of Diminished is in this test about 26 percent, a value which lies within the limits actually obtained in ordinary out-crosses of Diminished (see p. 64). And in the test of the Diminished mosaic the viability of the Diminished offspring was still more reduced by the simultaneous presence of numerous third-chromosome recessives which are expected to reduce the viability more in the Diminished than in the corresponding nonDiminished classes. From the numerical data of the case we arrive accordingly at the conclusion that one entire ovary in the mosaic was haplo-IV and the other diplo-IV, a conclusion which falls into line with the bilateral distribution of parts in the mosaic female.

In the test of the mosaic it was a striking fact that Diminished offspring did not emerge until the tenth day of counting. This result is exceptional, since in ordinary out-crosses of Diminished the first Diminished offspring generally appear on the third or fourth day and never so late as was here the case. This seems to indicate that the egg development or the egg laying is retarded in the Diminished phenotype so that in the mosaic the eggs from the normal ovary have been deposited earlier than those from the haplo-IV ovary. This in combination with the slow development typical of the Diminished zygote would account for the very late emergence of the Diminished offspring of the mosaic in the first test culture (C.4732 p. 67). Special tests in order to compare the time of egg laying in Diminished and in non-Diminished flies have not been carried out.

The fact that the mosaic female in the first culture bottle (C. 4732) gave 262 non-Diminished and only 16 Diminished flies, the first of these appearing on the tenth day of counting, while in the other culture (C. 4732a, p. 67) 131 non-Diminished and 15 Diminished flies were obtained, the first Diminished individuals here appearing on the fourth day, lends 
support to the above assumption. In this case it would be more correct to use the data from the ten days' counts of C. 4732a only for deductions concerning the constitution of the ovaries of the mosaic female.

Of the 131 non-Diminished offspring in C. 4732a one third of the 131 non-Diminished flies plus 15 Diminished individuals are expected to be derived from the haplo-IV ovary. This ratio, 44:15, corresponds to a viability of Diminished of 34 percent, a value that is in still better accordance with the known viability of Diminished in ordinary out-cross tests.

\section{SUMMARY}

The possibility of delivering the combined genetical and cytological proof of somatic elimination of a chromosome as a cause of mosaicism is discussed.

A female mosaic is described. The entire right half plus the ventral surface of the left first coxa and the left half of the fifth tergite were wildtype; the rest of the individual exhibited a typical "Minute" character complex. Except for the slight deviations mentioned, the distribution of Minute and of wild-type parts was strictly bilateral both on the dorsal and on the ventral surface.

The mosaic was fertile, and the Minute character complex reappeared unchanged in a proportion of the $F_{1}$ flies. By appropriate genetical tests it was demonstrated that this dominant Minute was not due to an ordinary gene but to deficiency for one member of the fourth chromosome pair.

Cytological examination of ovaries from the Minute females proved that their cells contained only one member of the fourth chromosome pair as had been predicted. Hence the Minute mutant here dealt with is identical with the Diminished (haplo-IV) type.

The evidence presented proves conclusively that the elimination of the fourth chromosome must have occurred in one of the earliest, in all probability the first, cleavage divisions of the egg from which the mosaic developed. And the case represents the first in which somatic elimination of a chromosome (resulting in mosaicism) has been proved by combined genetical analysis and cytological demonstration.

Based on the numerical data of the case it is concluded that in the mosaic one entire ovary was haplo-IV and the other diplo-IV. Hence the case involves genetical evidence to the effect that in Drosophila more than one cleavage cell goes into the formation of the germ tract.

Some data on special peculiarities of the black purple curved Diminished and the Diminished-eyeless phenotype are presented and discussed in the appendix. 


\section{LITERATURE CITED}

BRIDGES, C. B., 1917 Deficiency. Genetics 2 : 445-465.

1921 Genetical and cytological proof of non-disjunction of the fourth chromosome in Drosophila melanogaster. Proc. Nat. Acad. Sci. Wash. 7: 186-192.

DoBZHANSKy, $\mathrm{T}$., 1931 Interaction between female and male parts in gynandromorphs of Drosophila simulans. Arch. Entro Mech. Organ. 123:719-745.

HUetTner, A. F., 1923 The origin of the germ cells in Drosophila melanogaster. J. Morph. 37: 385-423.

MoHR, O. L., 1919 Character changes caused by mutation of an entire region of a chromosome in Drosophila melanogaster. Genetics 4:275-282.

1923 A somatic mutation in the singed locus of the $\mathrm{X}$ chromosome in Drosophila melanogaster Hereditas $4: 142-160$.

1929 Exaggeration and inbibition phenomena encountered in the analysis of an autosomal dominant. Z. indukt. Abstamm.- u. VererbLehre. 50: 113-200.

1930 Genetisk og cytologisk bevis for somatisk elimination av et kromosom. Avh. Norske Vid.-Akad., Mat.-Naturv. K1. No. 12: 1-9. (Also in Archives de Biologie, 1931.)

Morgan, L. V., 1929 Composites of Drosophila melanogaster. Pub. Carnegie Instn. Washington $399: 1-296$.

Morgan, T. H., and Bridges, C. B., 1919 The origin of gynandromorphs. Pub. Carnegie Instn. Washington 278: 1-122.

Morgan, T. H., Bridges, C. B., and Sturtevant, A. H., 1925 The genetics of Drosophila. Bibl. genet. 2: 1-262.

Patterson, J. T., and Muller, H. J., 1930 Are progressive mutations produced by X-rays? Genetics 15 : 495-577.

Pearson, N. E., 1927 A study of gynandromorphic Katydids. Amer. Nat. 61: 163-168.

STERN, C., 1927a Über Chromosomenelimination bei der Taufliege. Naturwissenschaften 15: $740-746$.

1927b Ein genetischer und zytologischer Beweis für Vererbung im Y-Chromosom von Drosophila melanogaster. Z. Indukt. Abstamm.-u. VererbLehre 44: 187-231.

StuRTevant, A. H., 1929 The claret mutant type of Drosophila simulans: A study of chromosome elimination and of cell lineage. Z. wiss. Zool. 135 : 325-356.

\section{APPENDIX}

\section{Notes on the black purple curved Diminished and the Di- minished-eyeless phenotype}

In the male backcross of Diminished flies heterozygous for the II-chromosome recessives black purple and curved, there was a very extreme lowering of the viability of the $b p_{r} c D_{m}$ class (C. 4798-99, p. 69), and the very few $b p_{r} c D_{m}$ flies obtained were all males. A certain lowering of the viability of the $b p_{r} c$ phenotype in comparison with the wild-type was also apparent in these cultures. But this fact seemed not sufficient to account for the very disproportionate lowering of the viability when $b p_{r} c$ flies were at the same time Diminished.

The male backcross was accordingly repeated (C. 4959). Though not quite as marked, the result was similar, the following offspring being obtained: $+90, b p_{r} c 77, D_{m} 20, b p_{r} c D_{m} 4$ (all males). The latter individuals were small, weak and had a humid body surface so that they got stuck 
while emerging from the puparium. It was a general feature that the proximal tarsi were in these individuals very short and clumsy, while the distal ones were fused and reduced into a very thin thread without claws. In the forelegs these malformations caused irregularities in the sex combs which were also reduced in size. Though the tarsi are also in ordinary Diminished flies thinner than in the wild-type, these alterations in combination with the extreme lowering of the viability were so striking that the question arose whether we were here possibly dealing with exaggeration of non-included characters. In the few cases in which the wings of these $b p_{r} c D_{m}$ flies were unfolded, they were small, but otherwise like those of ordinary curved individuals.

A few tests were arranged in order to look into the matter. Diminished males heterozygous for purple were in two cultures backcrossed to purple females (C. 4975,5038 ). The output were in the two cultures: wild-type $66+59, p_{r} 64+49, D_{m} 32+71, p_{r} D_{m} 14+58$, respectively. In some young $p_{r} D_{m}$ individuals the $p_{r}$ character seemed perhaps to be slightly more transparent than in ordinary purple flies of the same age. But this seemed simply to be connected with the larger and more roundish eye form typical of the Diminished individuals. As seen from the totals, there was in one of the cultures a certain lowering of the viability of the $p_{r} D_{m}$ flies, but in the other, where the viability of Diminished was extraordinarily good, the $p_{r}$ $D_{m}$ class even outweighed the corresponding $p_{r}$ class.

Analogous tests were also carried out with curved (C. 5051-52) and gave: wild-type $96+62, c 92+80, D_{m} 80+79, c D_{m} 49+31$. The curved character failed to show any modification in combination with Diminished. The tarsi were normal. But it will be noticed that there is a distinct lowering of the viability of the $c D_{m}$ phenotype, although this lowering is of an entirely different order from that typical of the compounds of Diminished with the included IV-chromosome recessives.

An $\mathrm{F}_{2}$ was also raised from a female heterozygous for $b p_{r} c$ by males which in addition were Diminished with the following result (C. 4977): $+116, b p_{r} c 47 ; b 6, p_{r} c 3 ; b p_{r} 5, c 7 ; b c 2, p_{r} 1 ; D_{m} 71, b p_{r} c D_{m} 3 ; b D_{m} 4$; $b p_{r} D_{m} 1 ; c D_{m} 5$. The $b p_{r} c D_{m}$ flies, all males, had thread-like fused tarsi and got stuck while emerging. The other $D_{m}$ classes showed no disproportionate modifications. Finally, two black Diminished males from C. 4977 were mated to $b p_{r} c$ females and gave (C. 5008): $b 106, b p_{r} c 121, b D_{m} 41$, $b p_{r} c D_{m} 3$ (all males of the type described). The $b D_{m}$ flies were slightly lighter and more brownish than ordinary black individuals, but not more so than would be expected in view of the light body color typical of $\mathrm{Di}$ minished.

Genetics 17： Ja 1932 
Judging from the above tests the very low viability of the $b p_{r} c D_{m}$ phenotype must be due to an additive effect of the lowering of the viability caused by these II-chromosome recessives separately in combination with Diminished. And the disproportionate modification of the $b p_{r} c D_{m}$ phenotype must correspondingly be due to a summation of latent tendencies which are not by themselves strong enough to produce manifest character changes. In view of such evidence a certain caution seems advisable when it is a question of considering lowering of the viability as evidence of exaggeration.

The Diminished-eyeless compound described above (p. 71) contrasts with other typical cases of exaggeration in so far as the exaggeration of the eyeless character is almost negligible in spite of the very extreme lowering of the viability of the compound. This situation seems still more surprising if PATterson and MULler (1930) are right in considering a new IV-chromosome mutation which reduces the size of the eyes as a dominant allelomorph of eyeless. In this case "dominant eyeless" would, according to the statements given, have to be considered as a more extreme allelomorph than ordinary eyeless. In view of the best analyzed cases of exaggeration we would then have expected more extreme character changes, analogous to those present in "dominant eyeless," also in the Diminished-eyeless compound, since the analysis of the other cases of deficiency has led to the conclusion that deficiency for a given locus represents the final step, the bottom, so to speak, of the series of mutative changes that are possible in this particular locus (see on this point MOHR 1929).

However, the evidence for "dominant eyeless" being an ordinary point mutation allelomorphic to eyeless seems not fully convincing, and PATterSON and MULLER also express themselves with a certain reservation on this point. It is also possible that the apparent inconsistency mentioned above may bear relation to the fact that we are here dealing with a chromosome deficiency. It may well be that a more thorough comparison may show that the exaggeration phenomena in section deficiency and in chromosome deficiency though analogous in main features, may nevertheless show typical differences that are not yet recognized. 